\title{
The biogeography of the Plastisphere: implications for policy
}

\author{
Linda A Amaral-Zettler ${ }^{1,2^{*}}$, Erik R Zettler ${ }^{3}$, Beth Slikas ${ }^{1}$, Gregory D Boyd ${ }^{3}$, Donald W Melvin ${ }^{3}$, \\ Clare E Morrall ${ }^{4}$, Giora Proskurowski ${ }^{5}$, and Tracy J Mincer ${ }^{6 \dagger}$
}

Microplastics (particles less than $5 \mathrm{~mm}$ ) numerically dominate marine debris and occur from coastal waters to mid-ocean gyres, where surface circulation concentrates them. Given the prevalence of plastic marine debris (PMD) and the rise in plastic production, the impacts of plastic on marine ecosystems will likely increase. Microscopic life (the "Plastisphere") thrives on these tiny floating "islands" of debris and can be transported long distances. Using next-generation DNA sequencing, we characterized bacterial communities from water and plastic samples from the North Pacific and North Atlantic subtropical gyres to determine whether the composition of different Plastisphere communities reflects their biogeographic origins. We found that these communities differed between ocean basins - and to a lesser extent between polymer types - and displayed latitudinal gradients in species richness. Our research reveals some of the impacts of microplastics on marine biodiversity, demonstrates that the effects and fate of PMD may vary considerably in different parts of the global ocean, and suggests that PMD mitigation will require regional management efforts.

Front Ecol Environ 2015; 13(10): 541-546, doi:10.1890/150017

$\mathrm{P}$ ublic awareness of plastic marine debris (PMD) and its environmental impacts has intensified due in part to greater numbers of publications (both scholarly and popular) and large-scale policy recommendations on the subject. The Joint Group of Experts on the Scientific Aspects of Marine Environmental Protection (GESAMP) - an advisory body to the United Nations on the prevention, reduction, and control of the degradation of the marine environment - provided one such recommendation: "assessing the importance of plastics and microplastics as a vector for the transfer of organisms" (www.gesamp.org). Implicit in this challenge is the need to determine the distribution of organisms that colonize PMD over time and space: what organisms are living on these small pieces of plastic, and how does their biogeography vary locally, regionally, and globally? Because "microplastics" are defined as plastic particles less than $5 \mathrm{~mm}$, most organisms that colonize PMD are microscopic in scale and are best investigated at the genetic level through the use of modern molecular biology approaches.

Although diatoms and bacteria were noted in the first papers mentioning PMD (Carpenter and Smith 1972), the ecological importance of the "Plastisphere", the thin film of life that inhabits all plastic floating in the ocean, has only recently regained attention (Zettler et al. 2013;

${ }^{1}$ Josephine Bay Paul Center for Comparative Molecular Biology and Evolution, Marine Biological Laboratory, Woods Hole, MA * amaral @mbl.edu); ${ }^{2}$ Department of Earth, Environmental, and Planetary Sciences, Brown University, Providence, RI; ${ }^{3}$ Sea Education Association, Woods Hole, MA; ${ }^{4}$ St George's University, St George's, Grenada; ${ }^{5}$ School of Oceanography, University of Washington, Seattle, WA; ${ }^{6}$ Department of Marine Chemistry and Geochemistry, WHOI, Woods Hole, MA ${ }^{\dagger}$ (tmincer@whoi.edu)
Oberbeckmann et al. 2014). As annual plastic production continues to increase (299 million metric tons in 2015 (PlasticsEurope 2015]), citizens, government agencies, and the plastics industry are attempting to manage and reduce waste. However, a proportion escapes the waste stream and enters the open ocean, where it can reside for decades as small pieces in oceanic gyres (Figure 1a). Recent studies estimate peak concentrations of $>10^{5}$ and $>10^{6}$ pieces per square kilometer in the North Atlantic Subtropical Gyre (NASG) and in the North Pacific Subtropical Gyre (NPSG), respectively (Law et al. 2010, 2014). As a major source of marine pollution and invasive alien species - two important ocean health index criteria - PMD has joined the ranks of other ocean stressors, including climate change, ocean acidification, overfishing, and habitat destruction (Halpern et al. 2012).

As a common consumer material, plastic has existed only for about 60 years, with the first report of plastic in the oceans dating from 1972 (Carpenter and Smith 1972). As an artificial substrate, plastic affords a unique opportunity to study microbial biogeography in light of the finite timescale that PMD has existed in the ocean. There is ongoing debate on the importance and indeed even the existence of a biogeography for microbes (Caron 2009). Previously highlighted patterns in microbial richness (the number of species) sometimes contradict typical macroecological patterns, such as higher species richness in the tropics relative to temperate regions (Nemergut et al. 2013; Barberan et al. 2014). However, recent studies show that particulate substrates can act as microgeographic barriers to gene flow in types of bacteria that prefer growing attached to substrates rather than suspended in the water column (Yawata et al. 2014). The heavily 

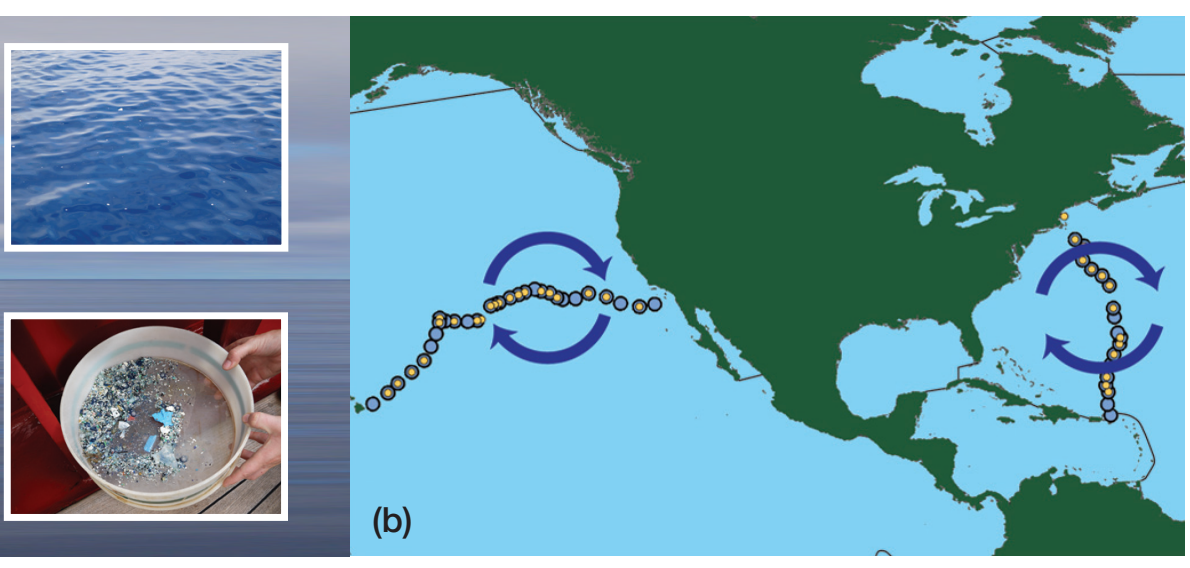

Figure 1. (a) The North Pacific "Garbage Patch" in the area with the highest concentration of plastic marine debris measured during the entire Pacific cruise. Even in this high-concentration area, which contains over 10 million pieces of plastic per square kilometer, the ocean appears to be relatively pristine unless one focuses carefully on the surface (upper inset). All samples are dominated by microplastics typically less than $5 \mathrm{~mm}$, as shown in the lower inset image of sieved plastic from the net tow at this station. (b) Map depicting locations where plastics and water samples from the two oceanic transects were collected for this study. Arrows indicate circulation of the North Atlantic and North Pacific Subtropical Gyres. Metadata and descriptions for all samples can be found in WebTable 1. Blue circles represent water samples only and yellow circles denote samples where both plastic and water were collected.

debated tenet that "everything is everywhere, but the environment selects" (Baas Becking 1934) argues for a Plastisphere community composition that should be homogeneous if it were heavily dependent on the nature of the plastic polymer itself as a substrate to colonize; however, "plastic" as a substrate can be immensely diverse in composition and chemical properties. If Plastisphere communities vary geographically, then there will be regional variation in the potential for the transport of invasive, harmful-algal-bloom-forming, and potentially pathogenic species across ocean basins, and in the interaction of microbes with plastics and their associated persistent organic pollutants, additives, and metals (Masó et al. 2003; Zettler et al. 2013). In trying to better understand this, several questions arise regarding the biogeography and assembly of Plastisphere communities. Do they reflect their terrestrial coastal origins - that is, are they influenced by the composition of the polymer type they colonize, or are they indicators of their regional surroundings? Do Plastisphere communities differ across oceanic biomes - eg the Atlantic versus Pacific? And do Plastisphere communities display the biogeographical patterns observed in macroecology, such as latitudinal gradients?

\section{Methods}

\section{Sample collection and plastic identification}

To determine the community structure of microbial organisms living on PMD and to test whether Plastisphere communities demonstrate biogeographical patterns, we collected PMD samples from a variety of marine environments, including a cruise track that extended from the tropics to temperate waters in the Atlantic and a transect running from California to
Hawaii in the Pacific (Figure 1b). We verified the composition of plastic polymer types using a combination of Raman spectroscopy and attenuated total reflection Fourier transform infrared (ATR-FTIR) spectroscopy. A PeakSeeker Pro Raman spectrometry system (Agiltron, Woburn, MA) with integrated microscope was used to generate spectra that were compared with reference scans from plastics of known composition using Agiltron RSIQ software. Resin identifications are shown in WebTable 1 under the "environmental material" field. We also investigated microbial colonization patterns by immersing sterile pieces of plastic not only in seawater samples collected from open-ocean sites but also in coastal waters along docks at three locations (Woods Hole, MA; San Diego, CA; and St George's, Grenada; see WebTable 1 and WebPanel 1 for details).

\section{DNA extraction, sequencing, and analysis}

Microbial molecular profiling based on the sequencing of short hypervariable regions of the conserved small subunit (16S) ribosomal RNA (rRNA) marker gene is now an established approach in microbial ecology for inventorying microbial diversity. Our choice of this variable region allows us to directly compare our results with the global database of sequence reads generated in a large number of studies - including one of our own, which examined a subset of the data used in the current study to ascertain dynamics of Vibrio oligotypes (species delineations based on small numbers of nucleotide variations along the 16S rRNA gene) in a heterogeneous array of environments (Schmidt et al. 2014). Counting the frequency of individual sequence reads provides a first-order description of the relative abundance of specific microbes 
(a)

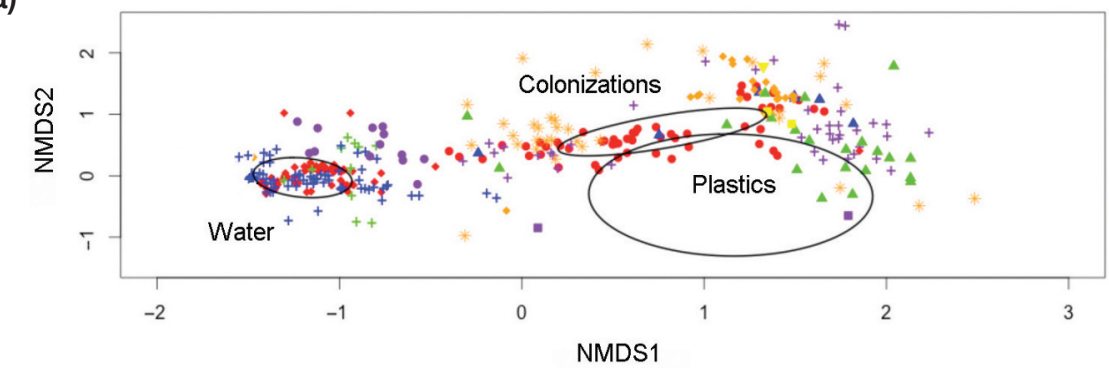

- Woods Hole coastal colonization

$\Delta$ Grenada coastal colonization

$\checkmark$ Pacific coastal colonization

- Atlantic open-ocean colonization

Caribbean macroplastic

+ Pacific microplastic

$\Delta$ Pacific macroplastic

- Pacific surface water

+ Pacific deep water

* Atlantic microplastic

Atlantic macroplastic

+ Atlantic surface water

- Atlantic deep water
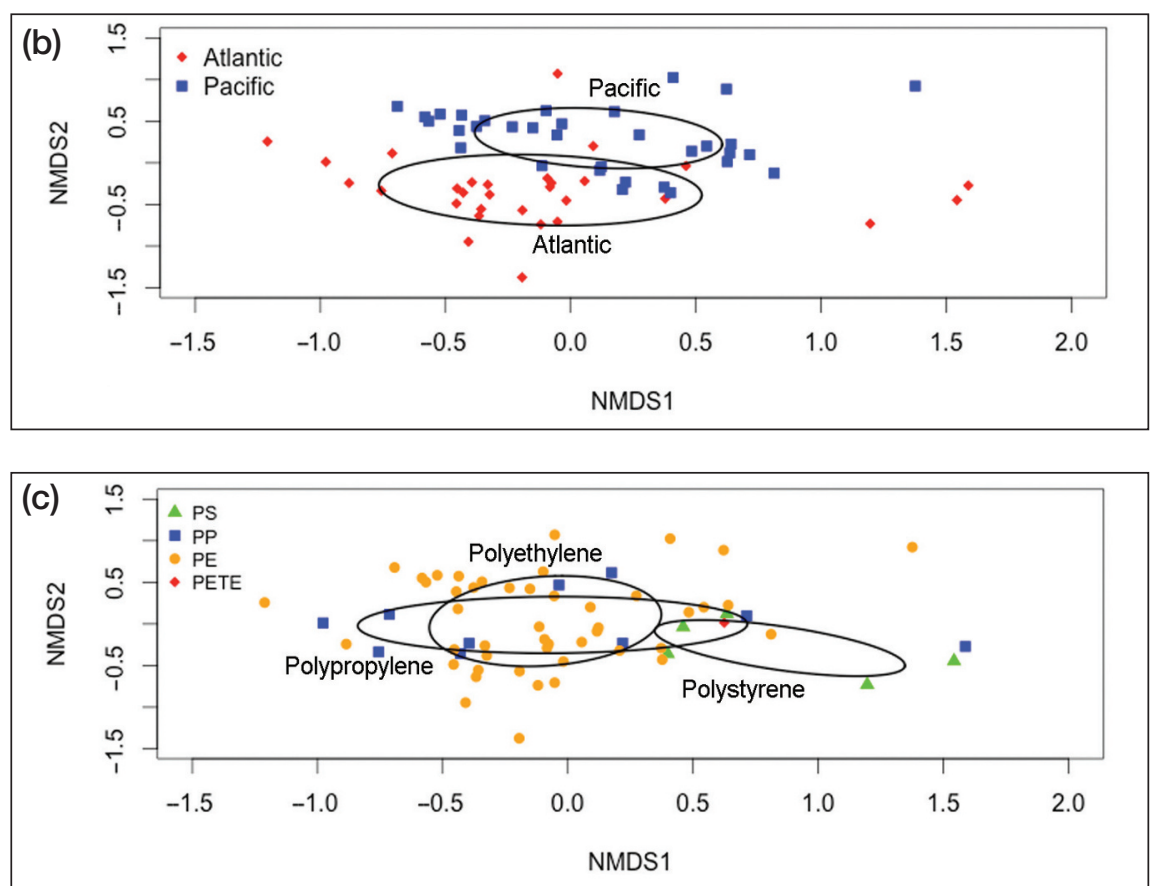

Figure 2. Nonmetric multidimensional scaling (NMDS) plots with dispersion ellipses showing the distinct clustering of microbial communities associated with (a) plastic exposed to coastal water at three docks and in the open ocean ("colonizations"), PMD collected in the field ("plastics"), and samples from the water column ("water") ( $\mathrm{k}=3$ axes; stress value $=0.11$ ). (b) NMDS plot differentiating Atlantic and Pacific microplastic populations with minimal overlap. (c) NMDS plot revealing the weak relationship between polymer type and associated microbial community similarities. Pairwise ANOSIM permutation tests (Table 1) revealed that samples were significantly differentiated by sample type and origin more than by polymer type. For the reduced dataset including only plastic samples ( $b$ and $c$ ), metaMDS was run with $\mathrm{k}=2$ axes, and the resulting stress value was 0.21 . $P S=$ polystyrene; $P P=$ polypropylene; $P E=$ polyethylene PETE = polyethylene terephthalate.

in a population. Comparing the resulting sequence data to that of published known taxa in public databases further refines taxonomic identification.

Total genomic DNA was extracted from all samples using a commercial kit (Gentra Puregene Kit, Qiagen, Valencia, CA) with modifications (WebPanel 1). DNA amplicon next-generation sequencing determined bacterial community profiles using the V6 hypervariable region of the 16S rRNA gene run on the Illumina HiSeq 1000 platform. All 346 samples were sequenced on five different Illumina HiSeq runs. A total of 209181709 reads passed our quality-filtering steps, with an average of
604571 reads per sample. Before analysis of the data, the number of reads per sample was normalized to the average value across all samples by random re-sampling. For those samples with less than the average number of reads, all reads were retained. The normalized dataset was clustered into 89865 clusters using the microbial comparison and analysis software package QIIME (Quantitative Insights Into Microbial Ecology; Caporaso et al. 2010), with a cluster similarity level of $97 \%$. Representative sequences were classified taxonomically using the Global Alignment for Sequence Taxonomy (GAST) algorithm (Huse et al. 2008) and an in-house database of archaeal, bacterial, and eukaryotic V6 sequences (refv6.tgz, http://vamps. mbl.edu/resources/databases.php). Nonmetric multidimensional scaling (NMDS) ordinations on standardized Bray-Curtis distance matrices were performed using the "vegan" package in $\mathrm{R}$ version 3.1 .3 and one-way Analysis of Similarity (ANOSIM) tests were performed in PRIMER version 6.1.16 (WebPanel 1). We estimated species richness using the Chao and Jost constant-coverage estimator (Chao and Jost 2012). See WebPanel 1 for more details on methods.

\section{Results}

Bacterial communities - those free-living in the water column versus those associated with PMD, and those colonizing plastic exposed to coastal versus open-ocean waters - were differentiated by NMDS ordinations and ANOSIM tests (Figure 2a; Table 1). When we compared bacterial communities based on oceanic biogeographic zone (Atlantic versus Pacific), ANOSIM permutation tests found significant distinct groupings (Global $R$ significance $=0.001)$. In contrast, pairwise tests of polymer 
types found significant differences only between polystyrene and polyethylene or between polystyrene and polypropylene (Table 1). We found that communities clustered more by geography (Figure $2 \mathrm{~b}$ ) than by polymer type (Figure 2c), with the exception of polystyrene as noted above. We also examined patterns of species richness along the latitudinal gradient from the Atlantic cruise transect, extending from tropical waters of the US Virgin Islands $\left(12.0^{\circ} \mathrm{N}\right)$ to the temperate waters off Cape Cod $\left(41.5^{\circ} \mathrm{N}\right)$ (Figure 1b; Figure 3, a-f). Estimated and observed bacterial richness exhibited clear latitudinal gradients in all three datasets (surface water, deep water, and plastic), with greater richness found at lower latitudes (the tropics). Our conventional inventory diversity assessment did not consider temporal scales of diversity (ie seasonal changes) along this transect - future studies may find this to be important in determining latitudinal gradients in microbial taxa on a fine scale.

\section{Discussion}

A previous study in northern European waters demonstrated spatial and temporal variation in the microbial communities associated with different plastic types (Oberbeckmann et al. 2014). As the first ocean-basinscale comparison of Plastisphere communities, our study shows that they are well-defined biogeographically, with distinct signatures in the Atlantic and Pacific Ocean Gyres, and exhibit a latitudinal gradient in species richness in the North Atlantic. Additionally, Plastisphere communities are genetically unique from the free-living water-column communities that envelop them and possess dominant taxa that are highly variable and diverse. Our finding that Pacific and Atlantic Plastisphere communities are distinct from each other has important implications for understanding microbial interactions with plastic and managing this pollutant on a global scale.

Table 1. Results from post-hoc analyses of ANOSIM tests

\begin{tabular}{|c|c|c|c|c|c|}
\hline Groups & R statistic & $\begin{array}{c}\text { Significance } \\
\text { level }\end{array}$ & $\begin{array}{c}\text { Possible } \\
\text { permutations }\end{array}$ & $\begin{array}{c}\text { Actual } \\
\text { permutations }\end{array}$ & $\begin{array}{c}\text { Number } \geq \\
\text { observed }\end{array}$ \\
\hline water, plastics & 0.826 & 0.001 & Very large & 999 & 0 \\
\hline water, colonizations & 0.858 & 0.001 & Very large & 999 & 0 \\
\hline plastics, colonizations & 0.475 & 0.001 & Very large & 999 & 0 \\
\hline PE, PP & 0.085 & 0.19 & Very large & 999 & 195 \\
\hline PE, PS & 0.318 & 0.02 & 2598960 & 999 & 19 \\
\hline PE, PETE & 0.099 & 0.271 & 48 & 48 & 13 \\
\hline PP, PS & 0.254 & 0.043 & 3003 & 999 & 42 \\
\hline PP, PETE & 0.093 & 0.273 & II & II & 3 \\
\hline PS, PETE & -0.24 & 0.833 & 6 & 6 & 5 \\
\hline PE, PP, PS, PETE & 0.167 & 0.037 & NA & 999 & 36 \\
\hline Atlantic, Pacific & 0.284 & 0.001 & NA & 999 & 0 \\
\hline water, plastics, colonizations & 0.777 & 0.001 & NA & 999 & 0 \\
\hline
\end{tabular}

Notes: Groupings with significant $R$ statistics are highlighted in bold. $\mathrm{PE}=$ polyethylene; $\mathrm{PP}=$ polypropylene; $\mathrm{PS}=$ polystyrene; $\mathrm{PETE}=$ polyethylene terephthalate. $\mathrm{NA}=$ global tests do not generate a value for possible permutations.
Microbial associations with PMD were noted in the first published report of microplastics in the ocean (Carpenter and Smith 1972). Microbes dominate the diversity and biomass of marine ecosystems, as well as controlling biogeochemical cycling in these systems. Despite this, very little is known about the communities of microbes that develop on PMD and how they interact with and transform plastic, one of the most common anthropogenic pollutants in the sea. Recognizing this knowledge gap, Harrison et al. (2011) called for research to determine spatiotemporal patterns of taxonomic composition and functional potential of plastic-colonizing microbes as critical to informing management decisions. However, the diversity of these communities, and how they compare to communities in the surrounding seawater, has only recently been studied using scanning electron microscopy; furthermore, relatively few samples have been tested using molecular biology tools (Zettler et al. 2013; Oberbeckmann et al. 2014). Our findings extend the understanding of this distinct and uniquely anthropogenic ocean habitat to the global scale, demonstrate important biogeographic patterns, and corroborate what has been observed regionally and extrapolated globally in the literature (Ladau et al. 2013): Plastisphere communities and the surrounding bacterial communities (both surface and deep water) exhibited higher species richness in the tropics in the Northern Hemisphere during the summer months.

Some plastics are created from toxic monomers (eg vinyl chloride), and many plastics contain plasticizers designed to modify the physical and chemical properties of the product - that are also toxic. Most compounds designated as "priority pollutants" under the US Clean Water Act readily attach to hydrophobic plastics in the ocean, to such an extent that Rochman et al. (2013) suggested that plastic waste be classified as hazardous waste. Despite plastic's hydrophobic nature, it is well established that marine microbes have overcome this potential barrier to colonization. Microbes have remarkable metabolic diversity and are known to degrade hydrocarbons, including plastic, as well as some of the priority pollutants associated with PMD. Our previous research has suggested that microbes are eroding pits into the surface of PMD, implying that they are contributing to the physical (if not chemical) degradation of the plastic.

Although microbes have settled onto the surfaces of $\mathrm{PMD}$, it remains to be determined whether individual Plastisphere taxa have adapted to this relatively new 


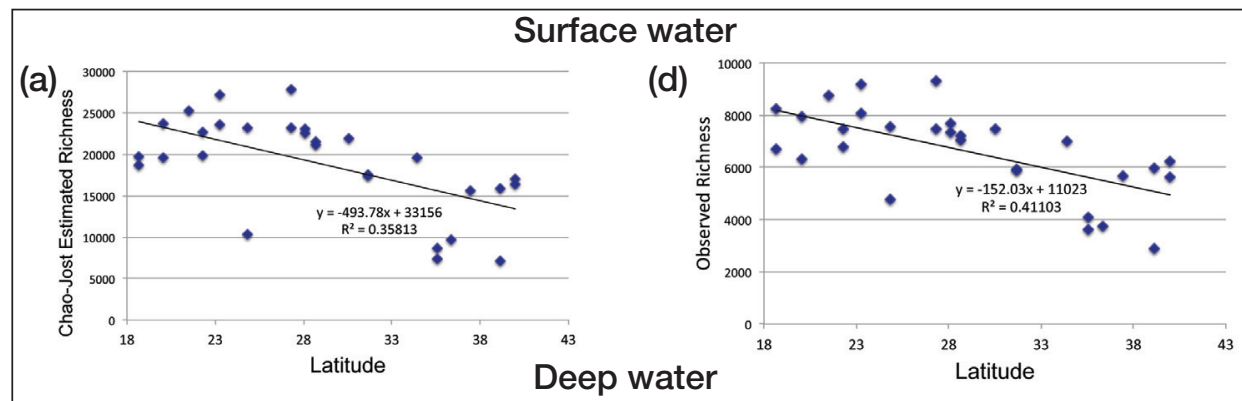

(b)

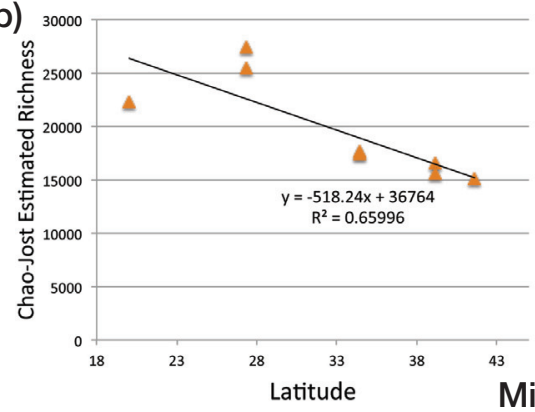

(e)

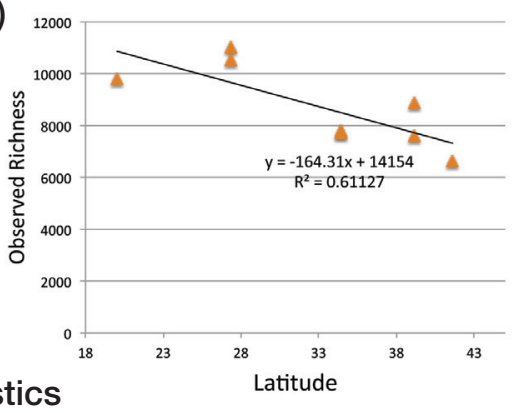

(c)

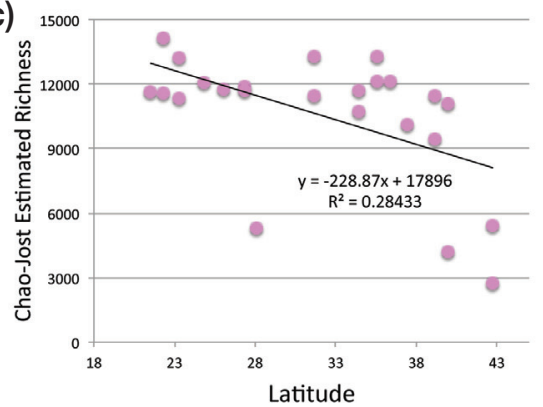

(f)

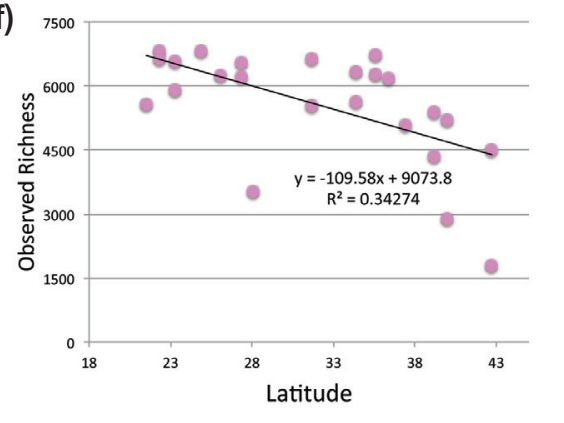

Figure 3. Estimated $(a-c)$ and observed $(d-f)$ species richness in bacterial operational taxonomic units (OTUs) along a latitudinal gradient in surface waters ( $a$ and $d ; \mathrm{P}<0.001$ ), in waters at $\sim 500-m$ depth ( $b$ and $e ; \mathrm{P}<0.05$ ), and on microplastics ( $c$ and $f ; \mathrm{P}<0.01$ ). Samples were collected May-June 2012, during a single cruise in the Atlantic; samples collected within $50 \mathrm{~km}$ of the coastline were excluded.

environmental niche. Based on the comparative diversity of communities we observed living on similar polymer substrates, Plastisphere communities appear to be highly variable in community dominance, as compared with the more stable populations in the surrounding seawater. Plastisphere biogeography may provide insights into the mechanisms driving the highly variable composition of microbial life on PMD. Attachment to PMD that drifts passively with ocean currents probably limits the active dispersal of PMD-associated microbes, thereby creating microniches in the environment, as has been shown on other particulate substrates (Finlay 2002; Wilkinson et al. 2012; Yawata et al. 2014). In contrast to microbes that "hitch rides" on actively dispersing organisms, microbes attached to microplastic marine debris passively float. Plastic is a long-lived substrate that can transport whole communities of microbes across ocean basins while contained in slow surface circulation patterns. The relative contribution of stochastic and deterministic processes in shaping the structure and composition of the Plastisphere requires further research.
There may also be a role for genetic drift in shaping the dominance structure of the Plastisphere community. Although most taxa in the Plastisphere are in low abundance, Plastisphere communities in general display a more even distribution than those in the surrounding seawater (Zettler et al. 2013). The role of disturbance in shaping Plastisphere communities should also be considered. Many organisms, from barnacles to whales, ingest plastic either accidentally or selectively, and the impact of ingestion as a disturbance could be important in restructuring microbial communities. The biofilm-coated nature of the Plastisphere likely makes these pieces of PMD - which may smell, taste, and look like food items - attractive to selective grazers, particularly those feeding in the oligotrophic (low-nutrient) ocean where food resources are scarce. This was illustrated in a study of loggerhead sea turtles (Caretta caretta) that selectively ingested white-colored plastic debris (Derraik 2002 and citations therein). PMD also serves as a mechanism for the persistence and spread of potential pathogens, such as Vibrio bacteria, which can sometimes dominate Plastisphere communities (Zettler et al. 2013). A piece of plastic passing through an animal's gut remains intact and, when excreted, provides a pulse of nutrients to the Plastisphere community in the oligotrophic ocean environment, particularly to opportunistic pathogens like Vibrio bacteria that survive the digestive tract environment. In addition, PMD serves to concentrate microbes that may require a minimum number of cells to infect a host and express pathogenicity genes. However, we know that these communities are subject to grazing not just by vertebrates but also by predatory bacteria, ciliates, and other grazers that all take part in transforming community composition. Ingestion, as a form of disturbance at different scales, may be resetting colonization by the Plastisphere community. This would short-circuit the role of evolutionary diversification (or gradual changes in community structure over long timescales) and dispersal in shaping resultant Plastisphere communities, thereby contributing to the observed variation in community composition.

Managing the threat of PMD, a potential that is attracting increasing interest, must be informed by 
research that targets policy-relevant issues. PMD is undoubtedly recognized as a global marine problem. However, efforts to monitor the environmental impacts of PMD must consider that distinct Plastisphere communities can occur at different locations and may have site-specific effects. For example, the call for including microplastic monitoring in existing regional and national programs and the adoption of global environmental standards should recognize the likely geographic variability in the underlying Plastisphere communities. Likewise, the European Union, which recommended that marine mammals be monitored for the composition and quantity of marine debris consumed, should consider where these organisms are feeding.

Among the GESAMP action-oriented recommendations was the need for "identifying the main sources and categories of plastics and microplastics entering the ocean". Our study revealed that regardless of point of entry, microbes that inhabit the Plastisphere tended to reflect their local surroundings more than their potential origins (ie coastal or terrestrial environments). One challenge in the interpretation of our data is that the age of PMD is difficult to determine. PMD age itself may play a part in shaping the underlying microbial community; however, it is unlikely that this is a major factor in source population relationships. Determining the longevity of different types of PMD in marine systems - along with the need to better characterize microbial interactions with the persistent, bioaccumulating, and toxic substances contained on it - represents future challenges in studying the relationships between PMD and the Plastisphere.

\section{Acknowledgements}

This work was supported by a US National Science Foundation (NSF) collaborative grant to LAA-Z (OCE-1155571), ERZ (OCE-1155379), and TJM (OCE-1155671), and was partially funded by an NSF TUES grant (DUE-1043468) to LAA-Z and ERZ, and by the Richard Saltonstall Charitable Foundation to TJM. GP was funded through the OCE-1155379 grant and assisted with identification of plastic resins via ATR-FTIR. Students and staff of SEA Semester academic programs (www.sea.edu) collected all open-ocean samples aboard the vessels SSV Corwith Cramer (Atlantic) and SSV Robert C Seamans (Pacific). We thank C Rochman for her donation of a Pacific plastic incubation sample. Sequence data from this study have been deposited in the National Center for Biotechnology Information's Sequence Read Archive under accession number SRP026054. See WebPanel 2 for author contributions.

\section{References}

Baas Becking L. 1934. Geobiologie of inleiding tot de milieukunde. The Hague, the Netherlands: WP Van Stockum \& Zoon.

Barberan A, Casamayor EO, and Fierer N. 2014. The microbial contribution to macroecology. Front Microbiol 5: 203.

Caporaso JG, Kuczynski J, Stombaugh J, et al. 2010. QIIME allows analysis of high-throughput community sequencing data. Nature Methods 7: 335-36.

Caron DA. 2009. Past President's address: protistan biogeography: why all the fuss? J Eukaryot Microbiol 56: 105-12.

Carpenter EJ and Smith Jr KL. 1972. Plastics on the Sargasso Sea surface. Science 175: 1240-41.

Chao A and Jost L. 2012. Coverage-based rarefaction and extrapolation: standardizing samples by completeness rather than size. Ecology 93: 2533-47.

Derraik J. 2002. The pollution of the marine environment by plastic debris: a review. Mar Pollut Bull 44: 842-52.

Finlay BJ. 2002. Global dispersal of free-living microbial eukaryote species. Science 296: 1061-63.

Halpern BS, Longo C, Hardy D, et al. 2012. An index to assess the health and benefits of the global ocean. Nature 488: 615-20.

Harrison JP, Sapp M, Schratzberger M, and Osborn AM. 2011. Interactions between microorganisms and marine microplastics: a call for research. Mar Technol Soc J 45: 12-20.

Huse SM, Dethlefsen L, Huber JA, et al. 2008. Exploring microbial diversity and taxonomy using SSU rRNA hypervariable tag sequencing. PLoS Genet 4: e1000255.

Ladau J, Sharpton TJ, Finucane MM, et al. 2013. Global marine bacterial diversity peaks at high latitudes in winter. ISME J 7: 1669-77.

Law KL, Moret-Ferguson SE, Goodwin DS, et al. 2014. Distribution of surface plastic debris in the Eastern Pacific Ocean from an 11-year data set. Environ Sci Technol 48: 4732-38.

Law KL, Moret-Ferguson S, Maximenko NA, et al. 2010. Plastic accumulation in the North Atlantic Subtropical Gyre. Science 329: $1185-88$.

Masó M, Garces E, Pages F, and Camp J. 2003. Drifting plastic debris as a potential vector for dispersing Harmful Algal Bloom (HAB) species. Sci Mar 67: 107-11.

Nemergut DR, Schmidt SK, Fukami T, et al. 2013. Patterns and processes of microbial community assembly. Microbiol Mol Biol $R$ 77: 342-56.

Oberbeckmann S, Loeder MGJ, Gerdts G, and Osborn AM. 2014. Spatial and seasonal variation in diversity and structure of microbial biofilms on marine plastics in Northern European waters. FEMS Microbiol Ecol 90: 478-92.

PlasticsEurope. 2015. Plastics - the facts 2014/2015. An analysis of European plastics production, demand and waste data. www. plasticseurope.org/documents/document/20150227150049. final_plastics_the_facts_2014_2015_260215.pdf. Viewed 31 Aug 2015.

Rochman CM, Browne MA, Halpern BS, et al. 2013. Policy: classify plastic waste as hazardous. Nature 494: 169-71.

Schmidt VT, Reveillaud J, Zettler E, et al. 2014. Oligotyping reveals community level habitat selection within the genus Vibrio. Front Microbiol 5: 563.

Wilkinson DM, Koumoutsaris S, Mitchell EAD, and Bey I. 2012. Modelling the effect of size on the aerial dispersal of microorganisms. J Biogeogr 39: 89-97.

Yawata Y, Cordero OX, Menolascina F, et al. 2014. Competition-dispersal tradeoff ecologically differentiates recently speciated marine bacterioplankton populations. P Natl Acad Sci USA 111: 5622-27.

Zettler ER, Mincer TJ, and Amaral-Zettler LA. 2013. Life in the "Plastisphere": microbial communities on plastic marine debris. Environ Sci Technol 47: 7137-46. 